\title{
Screening of promising maize genotypes against maize weevil (Sitophilus zeamais Motschulky) in storage condition
}

\author{
${ }^{1}$ Ram B Paneru and ${ }^{2}$ Resham B Thapa \\ ${ }^{1}$ Entomology Division, Khumaltar, Lalitpur \\ ${ }^{2}$ Agriculture and Forestry University, Rampur, Chitwan, Nepal \\ *Corresponding author email: rbpaneru10@gmail.com \\ ORCID ID:https://orcid.org/0000-0003-0684-6739
}

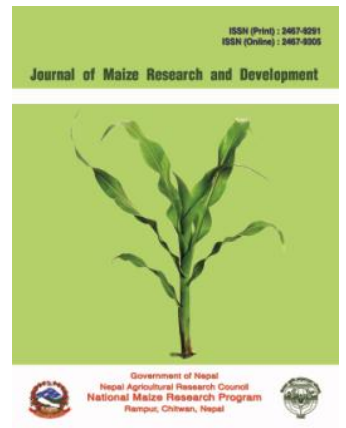

Received: August 27, 2017; Revised: September 19, 2017; Accepted: December 25, 2017

(C) Copyright 2017 Paneru and Thapa

\section{(1) (8)}

International License.

This work is licensed under a Creative Commons Attribution-NonCommercial 4.0

\begin{abstract}
The maize weevil (Sitophilus zeamais Motschulsky) is a serious pest of economic importance in stored grains. It causes major damage to stored maize grain thereby reducing its weight, quality and germination. An experiment was conducted in randomized complete block design (RCBD) with 3 replications to screen 32 maize genotypes against maize weevil in no-choice and free-choice conditions at Entomology Division, Khumaltar, Lalitpur (Room temperature: Maximum $24-32^{\circ} \mathrm{C}$ and Minimum $18-27^{\circ} \mathrm{C}$ ). The findings showed that the maize genotypes had different response to maize weevil damage ranging from susceptible to tolerance. The genotypes Manakamana-3, Lumle White POP Corn and Ganesh-2 showed their tolerance to S. zeamais as evidenced by lower number of weevil emerged/attracted, lower amount of grain debris release and lower proportion of bored grains, while the genotype ZM-627 was the most susceptible to weevil damage in both tests. The other remaining genotypes were intermediate types. This information is useful to improve grain protection in storage and varietal improvement/release program.
\end{abstract}

Keywords: Maize, genotype, storage, Sitophilus zeamais, damage, susceptible, tolerance

Correct citation: Paneru, R. B., \& Thapa, R.B. (2017). Screening of promising maize genotypes against maize weevil (Sitophilus zeamais Motschulky) in storage condition. Journal of Maize Research and Development, 3 (1),108-119. doi: http://dx.doi.org/10.3126/jmrd.v3i1.18927 
Journal of Maize Research and Development (2017) 3 (1):108-119

ISSN: 2467-9291 (Print), 2467-9305 (Online)

DOI: http://dx.doi.org/10.3126/jmrd.v3i1.18927

\section{INTRODUCTION}

Maize is one of the most important food crops in the world with the total production of 1070.5 million t., average yield of $5.96 \mathrm{t} / \mathrm{ha}$, occupying the second position (179.6 million ha) after wheat (220.8 million ha) (Statista, 2016/017). Maize is also the $2^{\text {nd }}$ most important cereal crop in Nepal in terms of area cultivated ( $891583 \mathrm{ha}$ ) and productivity ( $2.5 \mathrm{t} / \mathrm{ha})$ after rice (1362908 ha and 3.2 t/ha) (MoAD, 2015/016). This crop occupies about 26.9\% area of the total cereal cultivated, and contributes to about $25.7 \%$ of the total cereal production (AICC, 2016/017). Approximately, $73.5 \%$ of maize growing area lies in hilly regions followed by $16 \%$ in Terai and $10 \%$ in mountain domains (MoAD, 2012/013).

After harvest, maize is stored for household consumption, planting for the next season and for sale. But post harvest losses are high during maize storage in Nepal (Shivakoti \& Manandhar, 2000). Ghimire et al. (1996) indicated that the loss in maize grain weight was up to $20 \%$ in a typical post harvest storage situation, while KC (1992) reported 15-20\% post harvest losses in cereals. According to Sah (1998), weevil caused 51-97\% losses in the mid altitude (800-1500 masl) to low altitude ( $<800$ masl) irrespective of yellow or white maize when stored in a Kunew (maize cobs being heaped into a regular shape with no material supports inside the room) for a period of 5 months. In Nepal, maize grain loss due to weevil is high, and affordable alternatives to pesticides are inadequate. Majority of Nepalese farmers are illiterate and resources poor, therefore, they have no proper skills to acquire and handle synthetic pesticides. In such situation, search for effective and resistant/tolerant varieties are worthwhile without any cost to farmers. Insect resistant varieties offer greater advantages in developing countries where farmers can rarely afford to purchase insecticides for crop protection (Mihm, 1997). These varieties provide practical and economic way to minimize field and grain storage losses to improve both quantity and quality of stored grain for planting and human consumption (Simbaras et al., 2013). However, the level of varietal resistance or tolerance to weevil attack is not fully understood in Nepal. Hence, the present study was undertaken to screen different released/pipeline/promising maize genotypes against Sitophilus zeamais Motschulsky in storage.

\section{MATERIALS AND METHODS}

\section{Maize genotypes selection}

Maize genotypes (released/promising/pipelines) were collected from National Maize Research Program (NMRP), Rampur, Chitwan, Regional Agricultural Research Station (RARS), Lumle, and Agriculture Botany Division (ABD), Khumaltar for screening against S. zeamais (Table 1).

\section{Weevil inoculum}

Weevil culture was maintained in the Laboratory of Entomology Division, Khumaltar, Lalitpur to produce homogenous population ( $\mathrm{F}_{2}$-progeny) for the experiment. The male and female weevils were sexed as per Walker (2008) and Halstead (1963). 
Journal of Maize Research and Development (2017) 3 (1):108-119

ISSN: 2467-9291 (Print), 2467-9305 (Online)

DOI: http://dx.doi.org/10.3126/jmrd.v3i1.18927

Table 1. Maize genotypes selected for screening against S. zeamais in Khumaltar, Lalitpur, 2015

\begin{tabular}{clll}
\hline SN & Selected genotypes & Procured from & Remarks \\
\hline 1. & Rampur hybrid-2 & NMRP, Rampur & Hybrid maize \\
2. & RML 32/17 & NMRP, Rampur & Hybrid maize \\
3. & RML 4/17 & NMRP, Rampur & Hybrid maize \\
4. & RML 86/RML 96 & NMRP, Rampur & Hybrid maize \\
5. & Arun-2 & NMRP, Rampur & Early maturing maize \\
6. & Arun-4 & NMRP, Rampur & Early maturing maize \\
7. & Mankamana-3 & NMRP, Rampur & Normal season maize \\
8. & Across 99 42/Across 99 44 & NMRP, Rampur & Pipelines \\
9. & Across 9 331 RE & NMRP, Rampur & Pipelines \\
10. & Poshilo Makai-1 & NMRP, Rampur & Full season \\
11. & Poshilo Makai-2 (S99TLYQ-B) & NMRP, Rampur & Full season \\
12. & Rampur Composite & NMRP, Rampur & Full season \\
13. & ZM-401 & NMRP, Rampur & Pipelines \\
14. & TLBRS07F16 & NMRP, Rampur & Pipelines \\
15. & BGBYPOP & NMRP, Rampur & Pipelines \\
16. & ZM-627 & NMRP, Rampur & Pipelines \\
17. & 07SADVI & NMRP, Rampur & Pipelines \\
18. & 05SADVI & NMRP, Rampur & Pipelines \\
19. & P501SRCO/P502SRCO & NMRP, Rampur & Pipelines \\
20. & RML-95/RML-96 & NMRP, Rampur & Pipelines \\
21. & Mankamana-5 & RARS, Lumle & Normal season maize \\
22. & Mankamana-6 & RARS, Lumle & Normal season maize \\
23. & Lumle White POP Corn & RARS, Lumle & Promising line \\
24. & Lumle Yellow POP Corn & RARS, Lumle & Promising line \\
25. & Ganesh-2 & RARS, Lumle & Normal season maize \\
26. & Mankamana-1 & NMRP, Rampur & Normal season maize \\
27. & Khumal Yellow & ABD, Khumaltar & Normal season maize \\
28. & Deuti & ABD, Khumaltar & Normal season maize \\
29. & KSYM10 & NMRP, Rampur & Pipelines \\
30. & Mankamana-4 & ABD, Khumaltar & Normal season maize \\
31. & Pop Corn & ABD, Khumaltar & Pipelines \\
32. & Khumal hybrid-2 & ABD, Khumaltar & Hybrid maize \\
\hline
\end{tabular}

NMRP= National Maize Research Program, Rampur, Chitwan; RARS = Regional Agriculture Research Station; and $\mathrm{ABD}=$ Agri Botany Division, NARC, Nepal.

All the maize samples were oven dried at $130^{\circ} \mathrm{C}$ for $1 \mathrm{hr}$ to make them free from insects. The grain moisture content (GMC) of oven dried maize samples was determined by using a WILE Moisture Meter and then adjusted to $14 \%$ moisture for all the genotypes as per methods explained by Cecilia (1990). The experiment was conducted in free-choice and no-choice tests under the Laboratory condition (room temperature: Maximum $24-32^{\circ} \mathrm{C}$ and Minimum $18-27^{\circ} \mathrm{C}$ ) at Entomology Division, Khumaltar, Lalitpur from April to September, 2015.

\section{Free-choice test}

Thirty two maize genotypes of each $50 \mathrm{~g}$ grain samples were tested against $S$. zeamais. The experiment was set up in randomized complete block design (RCBD) in polythene bottle of $6 \mathrm{~cm}$ diameter and $7 \mathrm{~cm}$ height with 3 replications. Four circular holes were made at the bottom at 4 sides with no lid to allow weevils freely enter into the bottle. These bottles were placed in a 
Journal of Maize Research and Development (2017) 3 (1):108-119

ISSN: 2467-9291 (Print), 2467-9305 (Online)

DOI: http://dx.doi.org/10.3126/jmrd.v3i1.18927

circular manner inside a circular wide container $(50 \mathrm{~cm}$ diameter and $18 \mathrm{~cm}$ height) and 20 days old $800 \mathrm{~F}_{2}$-progeny of $S$. zeamais (irrespective of sexes) were released in the center. Then the wide container was covered with black muslin cloth. The experiment was set on first week and the first observation was taken for weevil attraction on last week of June, 2015. Subsequent observations were taken on last week of July, 2015 for progeny development and grain damage to each genotype.

\section{No-choice test}

For this test also, $50 \mathrm{~g}$ of maize samples were placed into a polythene bottle of $6 \mathrm{~cm}$ diameter and $7 \mathrm{~cm}$ height. Then 20 days old 5 pair of $\mathrm{F}_{2}$-progeny of $S$. zeamais (male and female) was introduced in each bottle as an inoculum. The mouth of bottles was perforated with black muslin cloth for free air circulation. All the bottles with maize samples along with weevil inoculums were placed inside the wide metal bins $(59 \mathrm{~cm}$ diameter and $33 \mathrm{~cm}$ height). The experiment was set on RCBD with 3 replications on the second week of July. The observations on number of progeny emerged, the quantity of grain debris released and the number of bored grains were recorded during the last week of August 2015.

Grains bored data were transformed into arcsine. Then data were analyzed using $\mathrm{R}$ package for analysis of variance and Duncan Multiple Range Test (DMRT) was used for significant mean separation at $5 \%$ level.

\section{RESULTS AND DISCUSSION}

The study showed that maize genotypes varied to response to maize weevil attack ranging from susceptible to tolerance, indicating the genotype resistance mechanisms.

\section{Effect of genotypes on number of S. zeamais progeny emergence}

In no-choice test, there were variations, and the significant differences were observed at $<1 \%$ level among the 32 genotypes for weevil progeny emergence (Table 2). It ranged from 67.3 to 300.3 mean adult emergence, which was low in Manakamana-3 followed by Lumle White POP Corn, Ganesh-2 and Rampur Composite indicating their tolerance to S. zeamais. Similarly, the mean number of weevil was high in RML 32/RML-17 followed by Poshilo Makai-2 (S99TLYQB), Poshilo Makai-1, Pop Corn and RML 86/RML 96 showing their susceptibility to $S$. zeamais. The remaining tested genotypes were intermediate types.

In free-choice test also, significant differences were observed at $<1 \%$ level among the tested genotypes (Table 2). It ranged from 136.7 to 220.7 mean weevil emergence. The mean number of progeny emergence was low in Ganesh-2 followed by RML-95/RML-96, Lumle Yellow POP Corn, Manakamana-1, Lumle White POP Corn, Manakamana-6 and Manakamana-3 indicating their tolerance to $S$. zeamais. Similarly, the mean number of progeny emergence was high in ZM-627, Across $9331 \mathrm{RE}$, Arun-4, TLBRS07F16, 05SADVI, RML 32/17 and P501SRCO/ P502SRCO showing their susceptibility to the S. zeamais. The rest of the genotypes were intermediate types. 
Journal of Maize Research and Development (2017) 3 (1):108-119

ISSN: 2467-9291 (Print), 2467-9305 (Online)

DOI: http://dx.doi.org/10.3126/jmrd.v3i1.18927

Table 2. Mean number of $S$. zeamais progeny emergence in selected maize genotypes at Khumaltar, Lalitpur, 2015

\begin{tabular}{|c|c|c|c|}
\hline \multirow[t]{2}{*}{$\mathrm{SN}$} & \multirow[t]{2}{*}{ Selected genotypes } & \multicolumn{2}{|c|}{ Mean number of weevil emergence $^{\circ}$} \\
\hline & & No-choice test & Free-choice test \\
\hline 1. & Rampur hybrid-2 & $143.7 \pm 16.7 \mathrm{~g}-\mathrm{j}$ & 195.7 \pm 17.0 a-h \\
\hline 2. & RML 32/17 & $300.3 \pm 30.2 \mathrm{a}$ & $210.7 \pm 0.3$ a-d \\
\hline 3. & RML 4/17 & $170.3 \pm 19.9 \mathrm{~b}-\mathrm{j}$ & $187.3 \pm 21.4 \mathrm{a}-\mathrm{i}$ \\
\hline 4. & RML 86/RML 96 & $216.7 \pm 24.5 \mathrm{~b}-\mathrm{e}$ & $208.7 \pm 29.7$ a-f \\
\hline 5. & Arun-2 & $190.3 \pm 10.7 \mathrm{~b}-\mathrm{i}$ & $190.3 \pm 30.7 \mathrm{a}-\mathrm{i}$ \\
\hline 6. & Arun-4 & $196.7 \pm 35.5$ b-h & $219.1 \pm 22.7 \mathrm{ab}$ \\
\hline 7. & Mankamana-3 & $67.3 \pm 11.9 \mathrm{k}$ & $158.7 \pm 26.8 \mathrm{e}-\mathrm{j}$ \\
\hline 8. & Across 99 42/Across 9944 & $191.0 \pm 1.0 \mathrm{~b}-\mathrm{i}$ & $163.7 \pm 6.4 \mathrm{c}-\mathrm{j}$ \\
\hline 9. & Across $9331 \mathrm{RE}$ & $202.3 \pm 23.3 \mathrm{~b}-\mathrm{g}$ & $220.3 \pm 23.1 \mathrm{ab}$ \\
\hline 10. & Poshilo Makai-1 & $234.0 \pm 20.2 \mathrm{abc}$ & $177.0 \pm 11.0 \mathrm{a}-\mathrm{j}$ \\
\hline 11. & Poshilo Makai-2 (S99TLYQ-B) & $242.0 \pm 1.7 \mathrm{ab}$ & $178.7 \pm 18.0 \mathrm{a}-\mathrm{j}$ \\
\hline 12. & Rampur Composite & $128.0 \pm 21.9 \mathrm{~h}-\mathrm{k}$ & $160.3 \pm 10.8 \mathrm{~d}-\mathrm{j}$ \\
\hline 13. & ZM-401 & $159.0 \pm 16.6 \mathrm{~d}-\mathrm{j}$ & $201.3 \pm 13.6 \mathrm{a}-\mathrm{g}$ \\
\hline 14. & TLBRS07F16 & $214.0 \pm 21.2 \mathrm{~b}-\mathrm{f}$ & $212.7 \pm 10.5 \mathrm{abc}$ \\
\hline 15 . & BGBYPOP & $188.3 \pm 20.2 \mathrm{~b}-\mathrm{i}$ & $168.3 \pm 12.7 \mathrm{~b}-\mathrm{j}$ \\
\hline 16. & ZM-627 & $207.0 \pm 35.6 \mathrm{~b}-\mathrm{g}$ & $220.7 \pm 12.2 \mathrm{a}$ \\
\hline 17. & 07SADVI & $166.7 \pm 27.9 \mathrm{c}-\mathrm{i}$ & $209.7 \pm 27.2$ a-e \\
\hline 18. & 05SADVI & $149.7 \pm 18.9 \mathrm{f}-\mathrm{j}$ & $214.3 \pm 24.8 \mathrm{abc}$ \\
\hline 19. & P501SRCO/P502SRCO & $192.7 \pm 6.7 b-h$ & $210.0 \pm 11.5 \mathrm{a}-\mathrm{d}$ \\
\hline 20. & RML-95/RML-96 & $155 \pm 17.1 \mathrm{f}-\mathrm{j}$ & $141.7 \pm 19.1 \mathrm{ij}$ \\
\hline 21. & Mankamana-5 & $176.7 \pm 19.6 \mathrm{~b}-\mathrm{j}$ & $173.7 \pm 17.1 \mathrm{a}-\mathrm{j}$ \\
\hline 22. & Mankamana-6 & $153.0 \pm 6.1 \mathrm{f}-\mathrm{j}$ & $156.3 \pm 19.9 \mathrm{f}-\mathrm{j}$ \\
\hline 23. & Lumle White POP Corn & $118.3 \pm 37.6 \mathrm{jk}$ & $153.3 \pm 15.6 \mathrm{~g}-\mathrm{j}$ \\
\hline 24. & Lumle Yellow POP Corn & $189.7 \pm 11.1 \mathrm{~b}-\mathrm{i}$ & $146.7 \pm 11.4 \mathrm{hij}$ \\
\hline 25. & Ganesh-2 & $121.0 \pm 18.6 \mathrm{ijk}$ & $136.7 \pm 10.3 \mathrm{j}$ \\
\hline 26. & Mankamana-1 & $176.7 \pm 14.2 b-j$ & $151.0 \pm 11.1 \mathrm{~g}-\mathrm{j}$ \\
\hline 27. & Khumal Yellow & $171.7 \pm 21.0 \mathrm{~b}-\mathrm{j}$ & $193.7 \pm 1$ a-h \\
\hline 28. & Deuti & $156.7 \pm 2.3 \mathrm{e}-\mathrm{i}$ & $170.0 \pm 5.1 \mathrm{a}-\mathrm{j}$ \\
\hline 29. & KSYM10 & $155.3 \pm 10.2 \mathrm{f}-\mathrm{j}$ & $195.7 \pm 15.0 \mathrm{a}-\mathrm{g}$ \\
\hline 30. & Mankamana-4 & $163.3 \pm 9.0 \mathrm{c}-\mathrm{j}$ & $163.7 \pm 2.6 \mathrm{c}-\mathrm{j}$ \\
\hline 31. & Pop Corn & $224.7 \pm 38.0 \mathrm{bcd}$ & $172.3 \pm 9.9 \mathrm{a}-\mathrm{j}$ \\
\hline 32. & Khumal hybrid-2 & $156.3 \pm 16.3 \mathrm{f}-\mathrm{j}$ & $185.0 \pm 9.50 \mathrm{a}-\mathrm{i}$ \\
\hline & F Value & 4.22 & 2.75 \\
\hline & Probability & $5.65 \mathrm{e}^{-07}$ & 0.000352 \\
\hline & $\mathrm{CV}$ & $20.31 \%$ & $2.86 \%$ \\
\hline & DMRT & $* * *$ & $* * *$ \\
\hline
\end{tabular}

Values are means of three replications; ${ }^{0}$ Means followed by the same letters within each column are not significantly different at $5 \%$ level by DMRT.

In both tests, the maize genotypes Manakamana-3, Lumle White POP Corn and Ganesh-2 were tolerant to $S$. zeamais attack and the genotype RML 32/17 was susceptible one.

\section{Effect of maize genotypes on grain debris release by $S$. zeamais}

In no-choice test, the maize genotypes were statistically significant at $1 \%$ level for grain debris release (Table 3 ). It ranged from $0.2 \mathrm{~g}$ to $0.7 \mathrm{~g}$ mean grain debris, which was low in Manakamana3 followed by Rampur Composite and Ganesh-2 indicating their tolerance to S. zeamais. Similarly, the mean amount of grain debris release was high in Poshilo Makai-1 followed by 
Journal of Maize Research and Development (2017) 3 (1):108-119

ISSN: 2467-9291 (Print), 2467-9305 (Online)

DOI: http://dx.doi.org/10.3126/jmrd.v3i1.18927

Poshilo Makai-2 (S99TLYQ-B, P501SRCO/P502SRCO and ZM-627 showing their susceptibility to $S$. zeamais. The remaining tested genotypes were intermediate types.

Under free-choice test, the maize genotypes were statistically significant at $1 \%$ level for grain debris release at 20 days, which ranged from 0.02 to $0.09 \mathrm{~g}$ (Table 3 ).

Table 3. Effect of maize genotypes on amount of grain debris release by $S$. zeamais in storage at Khumaltar, Lalitpur, 2015

\begin{tabular}{|c|c|c|c|c|}
\hline \multirow[t]{2}{*}{ SN } & \multirow[t]{2}{*}{ Selected genotypes } & \multicolumn{3}{|c|}{ Mean amount of grain debris $(\mathrm{g})$} \\
\hline & & $\begin{array}{c}\text { No-choice test at } \\
50 \text { days }\end{array}$ & $\begin{array}{c}\text { Free-choice at } 20 \\
\text { days }\end{array}$ & Free-choice at 50 days \\
\hline 1. & Rampur hybrid-2 & $0.37 \pm 0.10 \mathrm{~b}-\mathrm{e}$ & $0.04 \pm 0.01 \mathrm{~g}-\mathrm{m}$ & $0.48 \pm 0.06 \mathrm{c}-\mathrm{h}$ \\
\hline 2. & RML 32/17 & $0.53 \pm 0.09$ a-d & $0.05 \pm 0.00 \mathrm{c}-1$ & $0.44 \pm 0.05$ e-h \\
\hline 3. & RML 4/17 & $0.42 \pm 0.11 \mathrm{~b}-\mathrm{e}$ & $0.08 \pm 0.01 \mathrm{abc}$ & $0.69 \pm 0.12 \mathrm{ab}$ \\
\hline 4. & RML 86/RML 96 & $0.40 \pm 0.06 \mathrm{~b}-\mathrm{e}$ & $0.06 \pm 0.02 \mathrm{a}-\mathrm{h}$ & $0.37 \pm 0.11 \mathrm{f}-\mathrm{k}$ \\
\hline 5 . & Arun-2 & $0.46 \pm 0.04 \mathrm{a}-\mathrm{d}$ & $0.05 \pm 0.01 \mathrm{c}-\mathrm{k}$ & $0.39 \pm 0.11 \mathrm{e}-\mathrm{j}$ \\
\hline 6. & Arun-4 & $0.46 \pm 0.06 \mathrm{a}-\mathrm{d}$ & $0.08 \pm 0.01 \mathrm{a}-\mathrm{d}$ & $0.41 \pm 0.06 \mathrm{e}-\mathrm{j}$ \\
\hline 7. & Mankamana-3 & $0.21 \pm 0.03 \mathrm{e}$ & $0.04 \pm 0.01 \mathrm{~h}-\mathrm{m}$ & $0.34 \pm 0.05 \mathrm{~h}-\mathrm{k}$ \\
\hline 8. & Across 99 42/Across 9944 & $0.53 \pm 0.01 \mathrm{a}-\mathrm{d}$ & $0.07 \pm 0.01 \mathrm{a}-\mathrm{g}$ & $0.50 \pm 0.07 \mathrm{c}-\mathrm{f}$ \\
\hline 9. & Across $9331 \mathrm{RE}$ & $0.53 \pm 0.09 \mathrm{a}-\mathrm{d}$ & $0.09 \pm 0.01 \mathrm{ab}$ & $0.59 \pm 0.13 \mathrm{bcd}$ \\
\hline 10. & Poshilo Makai-1 & $0.66 \pm 0.01 \mathrm{a}$ & $0.04 \pm 0.01 \mathrm{f}-\mathrm{m}$ & $0.45 \pm 0.06 \mathrm{~d}-\mathrm{h}$ \\
\hline 11. & Poshilo Makai-2 (S99TLYQ-B) & $0.58 \pm 0.05 \mathrm{ab}$ & $0.05 \pm 0.01 \mathrm{c}-1$ & $0.45 \pm 0.10 \mathrm{~d}-\mathrm{h}$ \\
\hline 12. & Rampur Composite & $0.30 \pm 0.08 \mathrm{de}$ & $0.03 \pm 0.01 \mathrm{j}-\mathrm{m}$ & $0.29 \pm 0.07 \mathrm{jk}$ \\
\hline 13. & ZM-401 & $0.42 \pm 0.08 \mathrm{~b}-\mathrm{e}$ & $0.04 \pm 0.01 \mathrm{~g}-\mathrm{m}$ & $0.60 \pm 0.08 \mathrm{bc}$ \\
\hline 14. & TLBRS07F16 & $0.52 \pm 0.08 \mathrm{a}-\mathrm{d}$ & $0.06 \pm 0.01 \mathrm{a}-\mathrm{h}$ & $0.74 \pm 0.08 \mathrm{a}$ \\
\hline 15. & BGBYPOP & $0.48 \pm 0.05 \mathrm{a}-\mathrm{d}$ & $0.04 \pm 0.01 \mathrm{~g}-\mathrm{m}$ & $0.45 \pm 0.03 \mathrm{~d}-\mathrm{h}$ \\
\hline 16. & ZM-627 & $0.55 \pm 0.08 \mathrm{abc}$ & $0.06 \pm 0.01 \mathrm{~b}-\mathrm{i}$ & $0.53 \pm 0.10 \mathrm{cde}$ \\
\hline 17. & 07SADVI & $0.35 \pm 0.09 \mathrm{~b}-\mathrm{e}$ & $0.07 \pm 0.01$ a-e & $0.43 \pm 0.06 \mathrm{e}-\mathrm{i}$ \\
\hline 18. & 05SADVI & $0.38 \pm 0.02 \mathrm{~b}-\mathrm{e}$ & $0.09 \pm 0.01 \mathrm{a}$ & $0.52 \pm 0.07 \mathrm{cde}$ \\
\hline 19. & P501SRCO/P502SRCO & $0.58 \pm 0.06 \mathrm{ab}$ & $0.07 \pm 0.00 \mathrm{a}-\mathrm{f}$ & $0.57 \pm 0.05 \mathrm{bcd}$ \\
\hline 20. & RML-95/RML-96 & $0.37 \pm 0.06 \mathrm{~b}-\mathrm{e}$ & $0.05 \pm 0.00 \mathrm{~d}-1$ & $0.30 \pm 0.02 \mathrm{ijk}$ \\
\hline 21. & Mankamana-5 & $0.42 \pm 0.02 \mathrm{~b}-\mathrm{e}$ & $0.03 \pm 0.00 \mathrm{j}-\mathrm{m}$ & $0.34 \pm 0.07 \mathrm{~g}-\mathrm{k}$ \\
\hline 22. & Mankamana-6 & $0.41 \pm 0.08 \mathrm{~b}-\mathrm{e}$ & $0.03 \pm 0.00 \mathrm{j}-\mathrm{m}$ & $0.29 \pm 0.05 \mathrm{jk}$ \\
\hline 23. & Lumle White POP Corn & $0.41 \pm 0.05 \mathrm{~b}-\mathrm{e}$ & $0.04 \pm 0.00 \mathrm{~h}-\mathrm{m}$ & $0.35 \pm 0.04 \mathrm{~g}-\mathrm{k}$ \\
\hline 24. & Lumle Yellow POP Corn & $0.47 \pm 0.02 \mathrm{a}-\mathrm{d}$ & $0.03 \pm 0.00 \mathrm{klm}$ & $0.26 \pm 0.04 \mathrm{jk}$ \\
\hline 25. & Ganesh-2 & $0.32 \pm 0.09$ cde & $0.02 \pm 0.00 \mathrm{~m}$ & $0.24 \pm 0.03 \mathrm{k}$ \\
\hline 26. & Mankamana-1 & $0.50 \pm 0.11 \mathrm{a}-\mathrm{d}$ & $0.02 \pm 0.00 \mathrm{~lm}$ & $0.28 \pm 0.06 \mathrm{jk}$ \\
\hline 27. & Khumal Yellow & $0.45 \pm 0.09$ a-e & $0.06 \pm 0.03 \mathrm{~b}-\mathrm{j}$ & $0.48 \pm 0.03 \mathrm{c}-\mathrm{g}$ \\
\hline 28. & Deuti & $0.51 \pm 0.07 \mathrm{a}-\mathrm{d}$ & $0.03 \pm 0.00 \mathrm{klm}$ & $0.39 \pm 0.03 e-j$ \\
\hline 29. & KSYM10 & $0.41 \pm 0.08 \mathrm{~b}-\mathrm{e}$ & $0.05 \pm 0.00 \mathrm{e}-1$ & $0.39 \pm 0.05 e-j$ \\
\hline 30. & Mankamana-4 & $0.47 \pm 0.04 \mathrm{a}-\mathrm{d}$ & $0.03 \pm 0.00 \mathrm{i}-\mathrm{m}$ & $0.49 \pm 0.11 \mathrm{c}-\mathrm{g}$ \\
\hline 31. & Pop Corn & $0.54 \pm 0.17 \mathrm{a}-\mathrm{d}$ & $0.02 \pm 0.00 \mathrm{~lm}$ & $0.28 \pm 0.04 \mathrm{jk}$ \\
\hline 32. & Khumal hybrid-2 & $0.37 \pm 0.04 \mathrm{~b}-\mathrm{e}$ & $0.03 \pm 0.01 \mathrm{~lm}$ & $0.30 \pm 0.04 \mathrm{ijk}$ \\
\hline & F- value & 1.609 & 5.242 & 8.229 \\
\hline & $\mathrm{P}$-value & 0.0547 & $1.55 \mathrm{e}^{-08}$ & $1.50 \mathrm{e}^{-12}$ \\
\hline & $\mathrm{CV}$ & $28.38 \%$ & $31.37 \%$ & $17.67 \%$ \\
\hline & DMRT & $* *$ & $* * *$ & $* * *$ \\
\hline
\end{tabular}

Values are means of three replications; ${ }^{0}$ Means followed by the same letters within each column are not significantly different at $5 \%$ level by DMRT.

The amount of grain debris release was low in Ganesh-2 followed by Pop Corn, Mankamana-1, Khumal hybrid-2, Lumle Yellow POP Corn, Deuti, Mankamana-5, Mankamana-6, Rampur Composite, Manakamana-4, Lumle White POP Corn and Mankamana-3 showing their tolerance 
Journal of Maize Research and Development (2017) 3 (1):108-119

ISSN: 2467-9291 (Print), 2467-9305 (Online)

DOI: http://dx.doi.org/10.3126/jmrd.v3i1.18927

to $S$. zeamais. Similarly, the amount of grain debris release was high in 05SADVI followed by Across 9331 RE, RML 4/17, Arun-4, 07SADVI, P501SRCO/P502SRCO and Across 99 42/Across 9944 indicating their susceptibility to $S$. zeamais. The remaining genotypes were intermediate types.

In similar test at 50 days, statistically significant differences were observed at $1 \%$ level for all tested genotypes (Table 3 ). It ranged from $0.2 \mathrm{~g}$ to $0.7 \mathrm{~g}$ mean grain debris which was low in Ganesh-2, Lumle Yellow POP Corn, Pop Corn, Manakamana-1, ManaKamana-6, Rampur Composite, Khumal Hybrid-2, RML-95/RML-96 and Manakama-3 indicating their tolerance to S. zeamais. Similarly, mean grain debris release was high in TLBRS07F16, RML 4/17, ZM-401, Across $9331 \mathrm{RE}, \mathrm{P} 501 \mathrm{SRCO} / \mathrm{P} 502 \mathrm{SRCO}, \mathrm{ZM}-627$ and 05SADVI showing their susceptibility to $S$. zeamais. The remaining tested genotypes were intermediate types. In both tests, the genotypes Manakamana-3, Lumle White POP Corn, Khumal Hybrid-2, and Ganesh -2 showed their tolerance to S. zeamais and the genotypes RML 32/17, BGBYPOP and ZM-627 showed their susceptibility to $S$. zeamais.

\section{Effects of maize genotypes on grain damage by $S$. zeamais}

In no-choice test, statistically significant differences were observed at $1 \%$ level among 32 genotypes for proportion of bored grains (Table 4). It ranged from 39.1 to $92.4 \%$. The mean percent of holes was low in Manakamana-3 followed by Lumle White POP Corn and Khumal Hybrid-2 showing their tolerance to S. zeamais. Similarly, mean percent of bored grains was high in Poshilo Makai-1 followed by RML 32/17, RML 86/RML 96, BGBYPOP, Poshilo Makai-2 (S99TLYQ-B) and ZM-627 showing their susceptibility to S. zeamais. The remaining tested genotypes were intermediate types.

In free-choice test as well, statistically significant difference was observed among the tested genotypes. Mean percent grains bored was low in Pop Corn followed by Lumle Yellow POP Corn, Manakamana-3, Ganesh-2, Khumal Hybrid-2 and Lumle White POP Corn indicating their tolerance to S. zeamais. Similarly, mean proportion of holed grains was high in Across 99 42/ Across 99 44, 05SADVI, ZM-401, BGBYPOP, ZM-627, RML 4/17, TLBRS07F16, Across 9 331 RE, RML 32/17 and P501SRCO/P502SRCO indicating their susceptibility to $S$. zeamais. In both test, Manakamana-3, Ganesh-2, Khumal Hybrid-2, Lumle White POP showed their tolerance and the genotypes BGBYPOP, ZM-627 and RML 32/17 showed their susceptibility. 
Journal of Maize Research and Development (2017) 3 (1):108-119

ISSN: 2467-9291 (Print), 2467-9305 (Online)

DOI: http://dx.doi.org/10.3126/jmrd.v3i1.18927

Table 4. Effect of maize genotypes on grain damage by $S$. zeamais in storage at Khumaltar, Lalitpur, 2015

\begin{tabular}{|c|c|c|c|}
\hline \multirow[t]{2}{*}{$\mathrm{SN}$} & \multirow[t]{2}{*}{ Selected genotypes } & \multicolumn{2}{|c|}{ Mean bored grains $(\%) \pm \mathrm{SE}$} \\
\hline & & No-choice test & Free-choice test \\
\hline 1. & Rampur hybrid-2 & $77.35 \pm 9.28 b-f$ & $89.58 \pm 2.29 \mathrm{c}-\mathrm{h}$ \\
\hline 2. & RML 32/17 & $91.16 \pm 0.13 \mathrm{ab}$ & $94.62 \pm 0.94 \mathrm{a}-\mathrm{e}$ \\
\hline 3. & RML 4/17 & $75.47 \pm 8.95 b-f$ & $94.58 \pm 2.29 \mathrm{a}-\mathrm{d}$ \\
\hline 4. & RML 86/RML 96 & $84.54 \pm 4.32 \mathrm{a}-\mathrm{d}$ & $82.83 \pm 4.77 \mathrm{~g}-\mathrm{j}$ \\
\hline 5. & Arun-2 & $86.48 \pm 2.31 \mathrm{a}-\mathrm{d}$ & $82.29 \pm 3.87 \mathrm{f}-\mathrm{j}$ \\
\hline 6. & Arun-4 & $83.31 \pm 7.31 \mathrm{a}-\mathrm{d}$ & $86.85 \pm 1.90 e-j$ \\
\hline 7. & Mankamana-3 & $39.06 \pm 7.02 \mathrm{~g}$ & $71.01 \pm 6.43 \mathrm{kl}$ \\
\hline 8. & Across 99 42/Across 9944 & $86.28 \pm 0.41 \mathrm{a}-\mathrm{d}$ & $98.13 \pm 1.18 \mathrm{a}$ \\
\hline 9. & Across $9331 \mathrm{RE}$ & $87.37 \pm 1.19 \mathrm{a}-\mathrm{d}$ & $94.68 \pm 1.33 \mathrm{a}-\mathrm{d}$ \\
\hline 10. & Poshilo Makai-1 & $92.39 \pm 0.58 \mathrm{a}$ & $90.13 \pm 2.31 \mathrm{~b}-\mathrm{h}$ \\
\hline 11. & Poshilo Makai-2 (S99TLYQ-B) & $90.46 \pm 1.18 \mathrm{abc}$ & $87.65 \pm 2.05 \mathrm{~d}-\mathrm{i}$ \\
\hline 12. & Rampur Composite & $73.12 \pm 7.59 \mathrm{def}$ & $82.94 \pm 1.71 \mathrm{~g}-\mathrm{k}$ \\
\hline 13. & ZM-401 & $78.56 \pm 7.07 \mathrm{a}-\mathrm{f}$ & $96.12 \pm 0.44 \mathrm{ab}$ \\
\hline 14. & TLBRS07F16 & $88.01 \pm 2.15 \mathrm{a}-\mathrm{d}$ & $94.79 \pm 1.40 \mathrm{a}-\mathrm{d}$ \\
\hline 15. & BGBYPOP & $90.36 \pm 4.06 \mathrm{ab}$ & $96.01 \pm 0.31 \mathrm{abc}$ \\
\hline 16. & ZM-627 & $89.75 \pm 2.78 \mathrm{abc}$ & $94.42 \pm 2.43 \mathrm{abc}$ \\
\hline 17. & 07SADVI & $75.62 \pm 6.15 \mathrm{c}-\mathrm{f}$ & $93.36 \pm 2.02 \mathrm{a}-\mathrm{f}$ \\
\hline 18. & 05SADVI & $77.71 \pm 9.15 \mathrm{a}-\mathrm{f}$ & $97.41 \pm 1.14 \mathrm{a}$ \\
\hline 19. & P501SRCO/P502SRCO & $87.17 \pm 2.28$ a-d & $94.50 \pm 0.40 \mathrm{a}-\mathrm{e}$ \\
\hline 20. & RML-95/RML-96 & $72.73 \pm 6.46 \mathrm{def}$ & $83.13 \pm 1.71 \mathrm{~g}-\mathrm{j}$ \\
\hline 21. & Mankamana-5 & $87.59 \pm 3.59 \mathrm{a}-\mathrm{d}$ & $83.58 \pm 3.80 \mathrm{~g}-\mathrm{j}$ \\
\hline 22. & Mankamana-6 & $83.70 \pm 3.41$ a-e & $82.63 \pm 6.67 \mathrm{~g}-\mathrm{j}$ \\
\hline 23. & Lumle White POP Corn & $60.65 \pm 11.85 \mathrm{fg}$ & $78.75 \pm 4.20 \mathrm{i}-1$ \\
\hline 24. & Lumle Yellow POP Corn & $78.33 \pm 1.85 b-f$ & $69.24 \pm 0.64 \mathrm{~lm}$ \\
\hline 25. & Ganesh-2 & $75.48 \pm 5.55 c-f$ & $76.97 \pm 2.57 \mathrm{jkl}$ \\
\hline 26. & Mankamana-1 & $83.68 \pm 2.88$ a-e & $80.12 \pm 7.39 \mathrm{~h}-\mathrm{k}$ \\
\hline 27. & Khumal Yellow & $82.32 \pm 1.31$ a-e & $94.42 \pm 0.57$ a-e \\
\hline 28. & Deuti & $88.74 \pm 2.37 \mathrm{a}-\mathrm{d}$ & $90.63 \pm 1.61 \mathrm{~b}-\mathrm{g}$ \\
\hline 29. & KSYM10 & $80.05 \pm 2.10 \mathrm{a}-\mathrm{f}$ & $91.07 \pm 2.82 \mathrm{~b}-\mathrm{g}$ \\
\hline 30. & Mankamana-4 & $85.68 \pm 1.57$ a-d & $90.30 \pm 2.98 \mathrm{~b}-\mathrm{g}$ \\
\hline 31. & Pop Corn & $77.84 \pm 3.46$ b-f & $58.63 \pm 4.93 \mathrm{~m}$ \\
\hline 32. & Khumal hybrid-2 & $64.29 \pm 11.41 \mathrm{ef}$ & $77.27 \pm 9.13 \mathrm{i}-1$ \\
\hline & F-value & 3.466 & 11.52 \\
\hline & P-value & $1.32^{\mathrm{e}-0} 5$ & $5.90 \mathrm{e}^{-16}$ \\
\hline & $\mathrm{CV}$ & $10.37 \%$ & $5.65 \%$ \\
\hline & DMRT & $* * *$ & $* * *$ \\
\hline
\end{tabular}

Values are means of three replications; $\mathrm{SE}=$ Standard error; ${ }^{0}$ Means followed by the same letters within each column are not significantly different at $5 \%$ level by DMRT.

\section{Effect of maize genotypes on S. zeamais preference}

In free-choice test, there was a statistically significant difference at $1 \%$ level for the mean number of weevils attracted on tested genotypes at 20 days (Table 5). The mean number of weevils attracted to the different genotypes ranged 13.3 to 37.7. The preference was high in P501SRCO/P502SRCO followed by Arun-4, Poshilo Makai-1, Rampur Hybrid-2, Arun-2, RML 86/RML 96 and Pop Corn. Similarly, the preference was low in RML-95/RML-96, Deuti, BGBYPOP, RML 4/17, Manakamana-4, Khumal yellow, Khumal hybrid-2, Across 99 42/Across 99 44, Manakamana-3. The remaining tested genotypes were intermediate types. 
Journal of Maize Research and Development (2017) 3 (1):108-119

ISSN: 2467-9291 (Print), 2467-9305 (Online)

DOI: http://dx.doi.org/10.3126/jmrd.v3i1.18927

Table 5. Preference of S. zeamais at 20 days on selected maize genotypes in Khumaltar, Lalitpur, 2015

\begin{tabular}{|c|c|c|}
\hline SN & Selected genotypes & S. zeamais adults attracted $(\mathrm{No}) \pm \mathrm{SE}$ \\
\hline 1. & Rampur hybrid-2 & $33.0 \pm 5.13 \mathrm{abc}$ \\
\hline 2. & RML 32/17 & $20.3 \pm 4.91 \mathrm{~b}-\mathrm{f}$ \\
\hline 3. & RML 4/17 & $15.7 \pm 4.37$ ef \\
\hline 4. & RML 86/RML 96 & $30.0 \pm 1.15 \mathrm{abc}$ \\
\hline 5. & Arun-2 & $30.7 \pm 4.06 \mathrm{abc}$ \\
\hline 6. & Arun-4 & $34.7 \pm 7.75 \mathrm{ab}$ \\
\hline 7. & Mankamana-3 & $18.3 \pm 1.20 \mathrm{c}-\mathrm{f}$ \\
\hline 8. & Across 99 42/Across 9944 & $17.7 \pm 2.03 \mathrm{c}-\mathrm{f}$ \\
\hline 9. & Across $9331 \mathrm{RE}$ & $25.0 \pm 4.93 \mathrm{a}-\mathrm{f}$ \\
\hline 10. & Poshilo Makai-1 & $33.3 \pm 3.48 \mathrm{abc}$ \\
\hline 11. & Poshilo Makai-2 (S99TLYQ-B) & $28.3 \pm 3.18 \mathrm{a}-\mathrm{d}$ \\
\hline 12. & Rampur Composite & $33.0 \pm 5.21 \mathrm{~b}-\mathrm{f}$ \\
\hline 13. & ZM-401 & $19.3 \pm 3.18$ a-f \\
\hline 14. & TLBRS07F16 & $28.0 \pm 5.51$ a-e \\
\hline 15. & BGBYPOP & $15.7 \pm 5.04 \mathrm{f}$ \\
\hline 16. & ZM-627 & $28.0 \pm 2.08 \mathrm{a}-\mathrm{d}$ \\
\hline 17. & 07SADVI & $26.0 \pm 3.21 \mathrm{a}-\mathrm{e}$ \\
\hline 18. & 05SADVI & $21.7 \pm 2.40 \mathrm{a}-\mathrm{f}$ \\
\hline 19. & P501SRCO/P502SRCO & $37.7 \pm 0.02 \mathrm{a}$ \\
\hline 20. & RML-95/RML-96 & $13.3 \pm 3.28 \mathrm{f}$ \\
\hline 21. & Mankamana-5 & $20.3 \pm 1.20 \mathrm{a}-\mathrm{f}$ \\
\hline 22. & Mankamana-6 & $22.3 \pm 2.73 \mathrm{a}-\mathrm{f}$ \\
\hline 23. & Lumle White POP Corn & $21.3 \pm 4.37 \mathrm{a}-\mathrm{f}$ \\
\hline 24. & Lumle Yellow POP Corn & $18.7 \pm 2.03 \mathrm{~b}-\mathrm{f}$ \\
\hline 25. & Ganesh-2 & $19.0 \pm 2.08 \mathrm{~b}-\mathrm{f}$ \\
\hline 26. & Mankamana-1 & $23.3 \pm 3.53 \mathrm{a}-\mathrm{f}$ \\
\hline 27. & Khumal Yellow & $17.7 \pm 3.18 \mathrm{c}-\mathrm{f}$ \\
\hline 28. & Deuti & $13.7 \pm 4.18 \mathrm{f}$ \\
\hline 29. & KSYM10 & $21.7 \pm 5.90$ a-f \\
\hline 30. & Mankamana-4 & $15.0 \pm 2.65 \mathrm{def}$ \\
\hline 31. & Pop Corn & $30.0 \pm 3.79 \mathrm{abc}$ \\
\hline \multirow[t]{5}{*}{32.} & Khumal hybrid-2 & $17.3 \pm 1.76 \mathrm{c}-\mathrm{f}$ \\
\hline & F-value & 2.37 \\
\hline & F-test & 0.00194 \\
\hline & $\mathrm{CV}$ & $10.92 \%$ \\
\hline & DMRT & $* * *$ \\
\hline
\end{tabular}

Values are means of three replications; $\mathrm{SE}=\mathrm{Standard}$ error; ${ }^{0}$ Means followed by the same letters within each column are not significantly different at $5 \%$ level by DMRT.

This study focused to the number of progeny emergence, amount of grain debris release, proportion of bored grains, number of $S$. zeamais attracted as important indicators of a genotype's susceptibility to weevil attack. Abebe et al. (2009) reported that an increasing number of $\mathrm{F}_{1}$-progeny resulted in an increasing grain damageg and grain weight loss. They found the numbers of $F_{1}$-progeny, percent grain damage and grain weight loss positively related with the susceptibility index. Resistance in stored maize to insect attack has been attributed to physical factors, such as grain hardness, pericap surface texture, and nutritional factors, such as amylose, lipid and protein content (Dobie, 1974; Tepping et al., 1988) or non-nutritional factors, 
Journal of Maize Research and Development (2017) 3 (1):108-119

ISSN: 2467-9291 (Print), 2467-9305 (Online)

DOI: http://dx.doi.org/10.3126/jmrd.v3i1.18927

especially phenolic compounds (Serratos et al., 1987). The role phenolics play in resistance formation in these surface tissues may be both related to structural components and antibiosis factors (Arnason et al., 1993). For weevils, grain hardiness has been reported as the main resistance parameter (Bamaiyi et al., 2007). The difference in the number of weevil emerged showed that there existed variations in susceptibility to maize weevil attack among the genotypes. The genotypes which recorded the higher number of weevil progeny emergence indicated the higher susceptibility to maize weevil attack and this might have been due to lack of resistance mechanisms. The low weevil emergence in genotypes can be attributed to high mortality of parent weevils. These parent weevils might have died before laying eggs thus few progeny emerged. The low weevil emergence in the genotypes may possibly be attributed to absence of essential nutrients and unbalanced proportion of nutrients leading to the death of weevil larvae. The significant variation for number of weevils emerged among the varieties could be due to antibiosis effects in the genotypes leading to retarded development of weevil progeny and sometimes death of weevils before laying eggs. The lower amount of grain debris release could be due to resistance mechanisms in or on the grain which prevented weevil attack. Thus, greater amount of debris released indicates more susceptibility to weevil attack than other experimental genotypes. Simbaras et al. (2013) reported that resistance mechanisms could be in the form of deterrents which could be biochemical or morphological or a combination of both. Biochemical compounds in the form of phenolic amides, such as defeuroyl and dicoumaroyl may be antibiosis factors to the weevil. These phenolic compounds have been detected by fluorescence imaging technique, which clearly shows the phenolic barrier to insects in the outer tissues. It has also been reported that antibiotic effects increased restlessness of insects, which reduced feeding and could explain how grain debris were low among tolerance genotypes. He also noted that variation in maize genotypes was due to antibiosis. Less amount of grain debris release could be attributed to antixenosis mechanisms like a smooth pericarp, which could deter weevils from oviposition and feeding and also prevents mandibles from gripping maize kernels. The great variation observed in the germplasms evaluated forms a genetic resource base for further improvement to raise the levels of resistance to weevils while conserving the preferred traits. This variation on response to $S$. zeamais attack gives an evidence of genetic diversity existence hence a rich genetic resource base for breeding insect resistance. Present findings offer good opportunity to exploit the variability for reducing post harvest insect pest loss, varietal improvement and release through genetic improvement.

\section{CONCLUSIONS}

The findings showed that the maize genotypes had different response to S. zeamais attack from susceptible to tolerance level. The genotypes Manakamana-3, Lumle White POP Corn and Ganesh-2 showed their tolerance to $S$. zeamais as evidenced by less number of weevil emerged/attracted, low amount of grain debris release and low proportion of bored grains in both free-choice and no-choice tests. The genotypes ZM-627 was the most susceptible one to $S$. zeamais. The remaining tested genotypes were intermediate types. Hence, there is ample opportunity to explore and utilize such genotypes in post harvest insect pest management, maize breeding programs and varietal improvement/release. 
Journal of Maize Research and Development (2017) 3 (1):108-119

ISSN: 2467-9291 (Print), 2467-9305 (Online)

DOI: http://dx.doi.org/10.3126/jmrd.v3i1.18927

\section{ACKNOWLEDGEMENTS}

This work was supported by the Nepal Agricultural Research Council, Entomology Division, Khumaltar, Lalitpur Nepal (grant numbers 306-72/73/002). The authors would like to thank CIMMYT Nepal for facilitating this work. Thanks are extended to Research Director NARC for encouragement to continue specialized studies in storage insect pest.

\section{AUTHOR CONTRIBUTIONS}

R. B. P. conceived and designed the experiments, collected data, analyzed the data and wrote the paper, and R. B. T. revised the article for final approval of the version to be published.

\section{CONFLICTS OF INTEREST}

The authors declare that there are no conflicts of interest.

\section{REFERENCES}

AICC. (2016/017). Agriculture Diary 2017. Agriculture Information and Communication Centre. Ministry of Agriculture Development. Hariharbhawan, Pulchowk, Lalitpur, Nepal.

Statista. (2016/017). Worldwide production of grain in 2016/17 by type (in million metric tons). Retrieved from https://www.statista.com/statistics/263977/world-grain-production-bytype. Accessed: Aug. 2017.

Arnason, J.T., Lambert J.D.H., Gale, J., \& Mihm, J. (1993). Is quality protein maize more susceptible than normal varieties to attack by the maize weevil, Sitophilus zeamais. Postharvest Biol. Technol., 2, 349-358.

Bamaiyi, L.J., Dike, M.C., \& Onu, I. (2007). Relative susceptibility of some sorghum varieties to the rice weevil Sitophilus oryzae L. (Coleoptera: Curculionidae). J. Entomol., 4, 387-392.

Cecilia, D.R., Hurburgh, C.R. Lawrence, J.R., Johnson, A., \& Fox, S.R. (1990). Adjustment of maize quality data for moisture content. Cereal Chem., 67(3), 292-295.

Dobie, P. (1974). The laboratory assessment of the inherent susceptibility of maize varieties to post harvest infestation by Sitophilus zeamais Motsch. (Coleoptera: Curculionidae) infesting field corn. J. Entomol. Sci., 21, 367-375.

Ghimire, A.J., Khanal, R., \& Katuwal, K.B. (1996). Post harvest storage situation in the eastern hills and losses in grain quantity and quality of field crop of Nepal. In: PAC Working Paper, Pakhribas Agricultural Centre, Dhankuta, Nepal.

Abebe, F., Tefera, T., Mugo, S., Beyene, Y., \& Vidal, S. (2009). Resistance of maize varieties to the maize weevil Sitophilus zeamais (Motsch.) (Coleoptera: Curculionidae). African J. Biotech., 8 (21), 5937-5943.

Halstead, D.G.H. (1963). External sex differences in stored products Coleoptera. Bull. Entomol. Res., 54, 119-134.

KC, G. (1992). On farm level pre-harvest and post harvest food loss preventive system in Nepal. Proceeding of National Seminar on Issues and Constraints Related to Post Harvest Food Loss Management 27 - 29 April, 1992, Kathmandu, Nepal. 
Journal of Maize Research and Development (2017) 3 (1):108-119

ISSN: 2467-9291 (Print), 2467-9305 (Online)

DOI: http://dx.doi.org/10.3126/jmrd.v3i1.18927

Khanal, R., Duwadi, V.R., Neupane, P.R., \& Ghimire, A.J. (1990). A report on the effect of local available plant materials against Sitophillus oryzae in maize. Paper Presented at the Summer Crops Seed Workshop held at PAC, Dhankuta, Nepal.

Mihm, J.A. (eds.) (1997). Insect resistant maize. Recent Advances and Utilization: Proceedings of an International Symposium held at the International Maize and Wheat Improvement Center (Commit) 27 November - 3 December 1994, DF: CIMMYT, Mexico.

MOAD. (2015/016). Statistical Information on Nepalese Agriculture 2015/016. Government of Nepal, Ministry of Agricultural Development, Monitoring, Evaluation and Statistics Division, Agri Statistics Section, Singha Durbar, Kathmandu, Nepal. June, 2017.

MOAD. (2012/013). Statistical Information on Nepalese Agriculture 2012/013. Government of Nepal, Ministry of Agricultural Development, Monitoring, Evaluation and Statistics Division, Agri Statistics Section, Singha Durbar, Kathmandu, Nepal. June, 2013.

Paneru, R.B., Duwadi, V.R., Khanal, R., \& Bhattarai, M.R. (1996). Testing of the efficacy of some local materials against weevils in stored maize. In: PAC Working Paper 139. Pakhribas Agricultural Centre, Dhankuta, Nepal.

Paneru, R.B., KC, H.B., \& Pradhan, S.B. (2010). Screening of botanical products against storage grain pests in maize storage system of mid-hills condition of Nepal. Proceedings of $26^{\text {th }}$ National Summer Crop Workshop organized by Nation Maize Research Program, Rampur, Chitwan during March 2 -6, 2010 in Rampur, Chitwan, Nepal.

Sah, Y.P. (1998). Minimizing the losses in stored maize under farmers' storage condition in the eastern hills of Nepal. Nepal Agricultural Research Council (NARC), Agricultural Research Station, Pakhribas, Dhankuta, Nepal.

Serratos, A., Ranason, J., \& Nozzolillo, C. (1987). Factors contributing resistance to exotic maize populations to maize weevil, Sitophilus zeamais. J. Chem. Ecol. 13, 751-761.

Shivakoti, G.P., \& Manandhar, D.N. (2000). An overview of post harvest losses in maize in Nepal. In: Proceedings of a Working Group Meeting of the Hill Maize Research Project, (Manandhar, D.N., Ransom, J.K. \& Rajbhandari, N.P. (eds.), 25 - 27 September 2000, Khumaltar, Lalitpur, Nepal. pp. 6 - 9.

Shivakoti, G.P. (1984). Post harvest losses at storage due to stored grain pests of maize under different storage structures. The $11^{\text {th }}$ Summer Crops Workshop held at Parwanipur Agriculture Station, Parwanipur, Jan. 30 - Feb. 2, 1984. pp 28 - 39.

Simbaras, H.M., Chitambe, J., \& Goto, S. (2013). Screening of stored maize (Zea mais L.) varieties grain for tolerance against maize weevil Sitophilus zeamais (Motsch). Intl. J. Plant Res., 3(3), 17-22.

Tepping, P.W., Legg, D.E., Rodriguez, J.G., \& Ponelei, C.G. (1988). Influence of maize pericarp surface relief on the resistance of the maize weevil. J. Kansas Entomol. Soc., 61, 237241.

Upadhyaya, I. P. (1998). Study on storage of maize see under ambient conditions in few storage containers. Annual Report (1997/098). Agricultural Engineering Division, Nepal Agricultural Research Council, Khumaltar, Lalitpur, Nepal.

Walker, K. (2008). Rice weevil (Sitophilus oryzae). Pest and Diseases Image Library. http:// www.padil.gov.au/viewPestDiagnosticImages.aspx?id=675 